\title{
An Evaluation of Detect and Avoid (DAA) Displays for Unmanned Aircraft Systems: The Effect of Information Level and Display Location on Pilot Performance
}

\author{
Lisa Fern ${ }^{1}$ and R. Conrad Rorie ${ }^{2}$ \\ San Jose State University, Moffett Field, CA, 94035 \\ Jessica S. Pack ${ }^{3}$ \\ Infoscitex Corporation, Wright-Patterson AFB, OH, 45433 \\ R. Jay Shively ${ }^{4}$ \\ NASA Ames Research Center, Moffett Field, CA, 94035 \\ and \\ Mark H. Draper ${ }^{5}$ \\ Air Force Research Laboratory, Wright-Patterson AFB, OH, 45433
}

\begin{abstract}
A consortium of government, industry and academia is currently working to establish minimum operational performance standards for Detect and Avoid (DAA) and Control and Communications (C2) systems in order to enable broader integration of Unmanned Aircraft Systems (UAS) into the National Airspace System (NAS). One subset of these performance standards will need to address the DAA display requirements that support an acceptable level of pilot performance. From a pilot's perspective, the DAA task is the maintenance of self separation and collision avoidance from other aircraft, utilizing the available information and controls within the Ground Control Station (GCS), including the DAA display. The pilot-in-the-loop DAA task requires the pilot to carry out three major functions: 1) detect a potential threat, 2) determine an appropriate resolution maneuver, and 3) execute that resolution maneuver via the GCS control and navigation interface(s). The purpose of the present study was to examine two main questions with respect to DAA display considerations that could impact pilots' ability to maintain well clear from other aircraft. First, what is the effect of a minimum (or basic) information display compared to an advanced information display on pilot performance? Second, what is the effect of display location on UAS pilot performance? Two levels of information level (basic, advanced) were compared across two levels of display location (standalone, integrated), for a total of four displays. The authors propose an eight-stage pilot-DAA interaction timeline from which several pilot response time metrics can be extracted. These metrics were compared across the four display conditions. The results indicate that the advanced displays had faster overall response times compared to the basic displays, however, there were no significant differences between the standalone and integrated displays. Implications of the findings on understanding pilot performance on the DAA task, the development of DAA display performance standards, as well as the need for future research are discussed.
\end{abstract}

\footnotetext{
${ }^{1}$ Senior Research Engineer, Human Systems Integration, MS 262-2, AIAA Member.

${ }^{2}$ Research Associate, Human Systems Integration, MS 262-2, AIAA Member.

${ }^{3}$ Human Factors Research Scientist, 711 HPW/RHC1, Bldg 146.

${ }^{4}$ Research Engineer, Human Systems Integration, MS 262-3.

${ }^{5}$ Technical Advisor \& Principal Engineering Research Psychologist, 711 HPW/RHC1, Bldg 45.
} 


\section{Acronyms}

$\begin{array}{ll}\text { ATC } & =\text { Air Traffic Control } \\ C 2 & =\text { Control and Communications } \\ C P A & =\text { Closest Point of Approach } \\ C S D & =\text { Cockpit Situation Display } \\ D A A & =\text { Detect and Avoid } \\ F A A & =\text { Federal Aviation Administration } \\ G C S & =\text { Ground Control Station } \\ J A D E M & =\text { Java Architecture for DAA Modeling and Extensibility } \\ M A C S & =\text { Multi-Aircraft Control Station } \\ N A S & =\text { National Airspace System } \\ N M A C & =\text { Near Mid Air Collision } \\ S C-228 & =\text { RTCA Special Committee 228 Minimum Operational Performance Standards for UAS } \\ T S D & =\text { Tactical Situation Display } \\ U A S & =\text { Unmanned Aircraft Systems } \\ V S C S & =\text { Vigilant Spirit Control Station }\end{array}$

\section{Introduction}

U NMANNED Aircraft Systems (UAS) have flown in the National Airspace System (NAS) in a limited and restricted capacity since the $1990 \mathrm{~s}^{1}$. Much of the early UAS operations in the NAS were for public purposes such as military training and border security, and occurred in remote locations and/or special use airspace. However, there has been a recent spike in the demand to operate UAS more widely, for both expanded public operations as well as civil and commercial purposes; commercial photography and videography, cargo transportation and delivery, communications and broadcasting, disaster response, infrastructure monitoring and science are some of the currently proposed applications for UAS. This demand creates the need for new policies to support the broader and more integrated operation of UAS in the NAS at an acceptable level of safety. New UASspecific policies will specify the development of new technologies to meet new or existing regulations and standards, since current UAS technologies were not developed with compliance to existing airspace operations, procedures, regulations, or standards in mind. One major regulatory driver identified by the Federal Aviation Administration (FAA) UAS Roadmap ${ }^{1}$, is the development of minimum standards for Detect and Avoid (DAA) and Control and Communications (C2) systems, which are currently being addressed by RTCA Special Committee 228 (SC-228), Minimum Operational Performance Standards for Unmanned Aircraft Systems, a consortium of government, industry and academic subject matter experts ${ }^{2}$.

\section{A. Detect and Avoid (DAA)}

The DAA system is a critical functional area intended to provide the pilot-in-command with the ability to self-separate from (i.e., remain well clear of), and avoid collisions with, other aircraft. The DAA functionality is an alternate method of compliance to the "see and avoid" requirements specified by the Title 14 Code of Federal Regulations (14CFR) section $91.113^{3}$, for a pilot on board a manned aircraft to remain well clear of other aircraft. Thus, the DAA system is intended to compensate for the removal of the pilot from the cockpit of an aircraft to a control station on the ground. The DAA system notionally consists of both hardware and software components: a surveillance sensor (or suite of sensors), data fusion/correlation logic, threat detection and resolution logic, the display of traffic information and resolution logic, and control and navigation interfaces to execute a resolution maneuver ${ }^{2}$. Since the DAA system introduces both new equipment and new pilot tasks to the entire UAS system, new or revised operational rules and standards are needed to ensure that the system and its architectural components are designed to an appropriate level of system performance and design assurance in order to meet an airspace safety threshold ${ }^{2,4}$. A key assumption for the near term minimum operational performance standards currently being established, is that there is a pilot-in-the-loop with the DAA system that has "the ability to command, intervene, and/or reject DAA maneuvering under normal operating conditions," 2 . Under this pilot-in-the-loop assumption, the pilot-in-command will directly execute a resolution maneuver via the Ground Control Station (GCS), as opposed to having a self separation capability that can execute a resolution maneuver automatically (either with or without pilot permission and/or supervision). Thus, one subset of these performance standards will need to address the DAA display requirements that support an acceptable level of pilot performance. 


\section{DAA and Pilot Performance}

From a pilot's perspective, the DAA task is the maintenance of self separation and collision avoidance from other aircraft, utilizing the available information and controls within the GCS. The pilot-in-the-loop DAA task requires the pilot to carry out three major functions: 1) detect a potential threat, 2) determine an appropriate resolution maneuver, and 3) execute that resolution maneuver via the GCS control and navigation interface(s). The most critical safety measure of pilot performance on the DAA task is the number of violations of a specified separation boundary (or how close the ownship came to another aircraft). The specified separation boundary may be a self separation threshold, such as well clear, and/or a collision avoidance boundary, such as a Near Mid Air Collision (NMAC). (Typically, the self separation threshold is larger than the collision avoidance threshold and may serve to warn the pilot of a potential collision avoidance threshold violation.)

Another useful quantification of pilot performance is measured response. Measured response has been used to quantify the end-to-end response time for a UAS pilot to complete an Air Traffic Control (ATC) clearance ${ }^{5,6,7}$. By breaking down the end-to-end response into discrete stages, from the issuance of the clearance until the UAS completes the maneuver, it is possible to extract discrete response time metrics, such as the time required for a pilot to initiate a control input into the GCS, or the time required to complete a control input in the GCS. A study by Rorie and Fern', found these response time metrics to be sensitive to differences in GCS command and control input interfaces. Table 1 presents the six stages of pilot-ATC interaction, identified by Rorie and Fern, for a pilot responding to

Table 1. The six stages of pilot-ATC interaction for a pilot responding to an ATC clearance. Reprinted with permission from Rorie and Fern?

\begin{tabular}{|c|c|c|}
\hline Stage & Description & Example \\
\hline $\mathrm{T}_{0}$ & $\begin{array}{l}\text { Initial ATC } \\
\text { Transmission }\end{array}$ & $\begin{array}{l}\text { "HAWK21, turn left heading 1-2-0, } \\
\text { vectors for your descent" }\end{array}$ \\
\hline $\mathrm{T}_{1}$ & Pilot Reply & $\begin{array}{l}\text { "Turn left heading 1-2-0, } \\
\text { HAWK21" }\end{array}$ \\
\hline $\mathrm{T}_{2}$ & Pilot Initiates Edit & Pilot opens editing window \\
\hline $\mathrm{T}_{3 \mathrm{a}}$ & $\begin{array}{l}\text { Pilot Uploads } 1^{\text {st }} \\
\text { Edit }\end{array}$ & $\begin{array}{l}\text { Pilot incorrectly uploads } 110^{\circ} \mathrm{Hdg} \\
\text { to the aircraft }\end{array}$ \\
\hline $\mathrm{T}_{3 \mathrm{~b}}$ & $\begin{array}{l}\text { Pilot Uploads } \\
\text { Final Edit }\end{array}$ & $\begin{array}{l}\text { Pilot correctly uploads } 120^{\circ} \mathrm{Hdg} \text { to } \\
\text { the aircraft }\end{array}$ \\
\hline $\mathrm{T}_{4}$ & $\begin{array}{l}\text { UAS Completes } \\
\text { Maneuver }\end{array}$ & HAWK 21 reaches $120^{\circ} \mathrm{Hdg}$ \\
\hline
\end{tabular}
an ATC clearance.

Measured response could be adapted for the DAA pilot task by quantifying the end-to-end response time for a UAS pilot to complete a self separation or collision avoidance maneuver in response to an alert presented on the DAA display. Pilot response time is critical to the maintenance of safe separation from other aircraft - delayed pilot responses could result in delayed aircraft maneuvering, which in turn could cause violations of separation thresholds. A delayed response may be due to a pilot's inability to detect a threat, determine an appropriate resolution, or execute the resolution. Alternatively, the operational environment may constrain the amount of time that the pilot has to detect and/or respond to potential conflicts. Firstly, pilots may be constrained in when they can detect threats; late detections may result from encounter geometries (e.g., last minute changes in altitude that result in a late "pop-up" threat) and/or limited surveillance ranges for UAS sensors, especially sensors intended to detect non-cooperating aircraft in the airspace. Secondly, pilots may be constrained in when they can maneuver against threats. For example, ATC may find it unacceptable and disruptive for pilots to maneuver too early against a potential threat; thus, considerations for interoperability with ATC must be taken into account. Given potentially limited timelines, the DAA display will need to support timely pilot responses to potential threats.

Response time metrics extracted from a DAA task timeline could be compared across different DAA concepts, such as DAA displays. This type of analysis would provide insight into how various DAA concepts or features impact pilots' overall DAA task performance, as well as potential effects on the three separate DAA functions (i.e., detect, determine, and execute). In addition, display concepts or features that support faster pilot response could be identified.

2. DAA Displays

The DAA display plays a critical role in supporting pilot performance on the DAA task. New regulations will need to specify the minimum set of information required to achieve a minimum acceptable level of performance to meet an airspace safety threshold. The minimum requirements for the DAA pilot display could specify the presentation of a minimum set of informational elements such as: ownship and intruder parameters (e.g., heading, altitude, airspeed), conflict detection and alerting, and conflict resolution guidance. The minimum information 
requirements will need to be based on quantitative evidence of acceptable levels of pilot performance, such as those discussed above.

While there have been several recent human-in-the-loop evaluations of traffic displays for $\mathrm{UAS}^{8,9,10}$, most have focused on military airspace operations rather than civil airspace operations. In addition, only a limited number of studies have examined traffic displays in the context of specific information requirements and their effect on pilot performance; that is, most studies have examined how to present a pre-determined set of information rather than what specific information is required.

A recent study examined the minimum visual information requirements for a UAS DAA system by comparing four display categories with different, and progressively more, information: Position, Direction, Prediction, and Rate $^{11}$. The Position display provided the pilot participants with instantaneous intruder positions and included aircraft identification, range, bearing, relative altitude, absolute altitude and numeric range. The Direction display added directionality as well as a heading chevron, numeric heading, and a vertical trend arrow. The Prediction display added yellow and red alert color-coding, and 30 second dead-reckoning vector lines to intruders. Finally, the Rate display added ground speed, history trails, and climb/descent rates. This study found that the Prediction display performed as good as, or better, than the Position and Direction displays, and no different than the Rate displays across a number of performance and workload metrics. Perhaps most importantly, the Prediction display resulted in significantly less NMACs than the two lower level displays (Position and Direction), and was not significantly different from the Rate display. The authors concluded that the information included in the Prediction display was the minimum visual information required for a DAA pilot display.

A survey conducted by Draper, Pack, Darrah, Moulton, \& Calhoun ${ }^{12}$, found that the majority of pilots surveyed indicated that the following information should be present at all times on a DAA display: intruder identification, intruder location, intruder relative position, intruder threat/alert level, DAA task priorities and status, DAA maneuver recommendations, flight restrictions, weather, navigation data, and visual alerts.

While Friedman-Berg et al. ${ }^{11}$ and Draper et al. ${ }^{12}$ sought to establish a minimum information set, Bell $^{13}$ compared a basic display (similar to the Direction display described in the Friedman-Berg et al. study) to two "advanced" concepts: a display that depicted the relative Closest Point of Approach (CPA) between ownship and intruder, and a display that depicted ownship avoidance areas with polygon shapes. This study found no significant differences in the frequency of violations of the defined well clear threshold between the three display concepts, however, there was a significant difference in the duration of violation events - the basic display had significantly longer violation durations than both of the other two advanced displays.

Together, these three studies provide a starting point for the systematic evaluation of candidate DAA displays, specifically with respect to determining the minimum information requirements for such displays. In addition to specifying the required informational elements, DAA display standards could address the location of the pilot display within the GCS, for example, whether the DAA display can be a standalone, separate display, or whether it needs to be integrated into the moving map, or tactical situation display. Display location has the potential to significantly impact pilot performance in maintaining self separation and collision avoidance from other aircraft. A standalone display is considered a near term technology solution for existing UAS, because it is easier to develop, certify and field an independent, separate display compared to modify existing GCS software. However, the standalone display concept has some disadvantages. First, a standalone traffic display is unlikely to be integrated with the GCS command and control interface, thus a pilot would have to identify a potential threat and resolution maneuver, and then translate that to the command and control interface on another display in order to execute the maneuver, and confusion could arise if the displays are at different zoom levels or orientations (e.g., track up versus north up). In addition, a standalone display is likely to be produced by a different manufacturer than the GCS, which could result in inconsistencies in the presentation of similar information between the DAA and GCS displays. Finally, pilot response times could be slower as pilots have to switch attention (and possibly interaction) between two different displays (and control inputs). While a DAA display that was integrated into the GCS moving map could overcome several of these disadvantages, in addition to the large increase in overhead in terms of resources to develop, a major risk of an integrated display is increased clutter on the moving map display. Thus, display location in addition to minimum display information, are critical issues that need to be understood within the context of pilot performance on the DAA task.

The simulation experiment detailed in this paper is the first of several planned experiments intended to quantify the minimum operational performance standards for DAA displays to support safe operation of UAS in the NAS. The goal of the planned experiments is to systematically evaluate the effect of candidate DAA display concepts (e.g., information elements, maneuver guidance, location, etc.) on pilots' response time and ability to maintain safe separation from other aircraft. The purpose of the present study was to examine two main questions with respect to DAA pilot display considerations for self separation and collision avoidance. First, what is the effect of a basic or 
minimum information display compared to an advanced information display on pilot performance? Second, what is the effect of display location on UAS pilot performance? This paper focuses specifically on pilots' measured response, adapted from the pilot-ATC interaction timeline shown in Table 1, while responding to self separation and collision avoidance encounters. Santiago and Mueller ${ }^{4}$ report on well clear violation metrics for this experiment.

The authors proposed three main hypotheses with respect to these questions:

1) Displays with an advanced information set will have faster response times overall than those with a minimum information set given the additional information provided by advanced displays to help the pilot detect potential threats and determine appropriate resolutions.

2) Integrated displays will support faster response times than standalone displays given the extra time required by a standalone display to translate traffic information from one display into a maneuver response on another display.

3) Given the above hypotheses, an advanced, integrated display will have faster response times than all other displays.

\section{Method}

\section{A. Participants}

Twelve active duty RQ-4 pilots ( $M=39$ years of age) were recruited for this experiment. Participants had an average of 216 hours of experience flying UAS in combat and non-combat military operations. Eight of the participants had prior experience flying UAS in civil airspace, each with an average of 60 hours. A single retired ATC served as a confederate for the study.

\section{B. Simulation Environment \\ 1. Ground Control Station}

Participants were situated at a UAS GCS containing two pieces of software distributed across four separate monitors, the Vigilant Spirit Control Station (VSCS) and the Cockpit Situation Display (CSD). Figure 1 shows the UAS GCS display set up. VSCS, developed by the Air Force Research Laboratory, is a mature GCS operator interface designed to support the control of UAS and their associated payloads ${ }^{14}$. In this study, VSCS generated three separate pilot displays in the ground station: a Tactical Situation Display (TSD), a health and status panel, and a simulated out-the-window nose camera display. The TSD (shown in Fig. 2) served as the pilot's primary display, providing ownship and route information, a moving map, and navigation and control interfaces. The TSD supported two separate vehicle control interfaces. The first, a waypoint-editing interface, allowed pilots to modify the assigned altitude or location of any waypoint on their

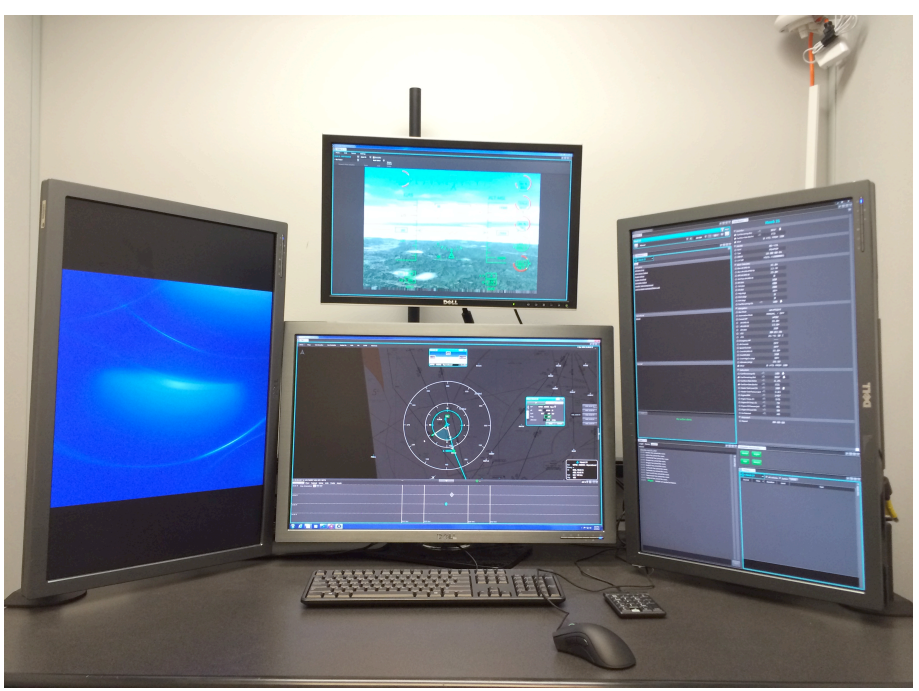

Figure 1. UAS GCS. CSD (not displayed), bottom left; TSD, bottom center; out-the-window view, top center; health and status panel, bottom right. mission route. The second, an autopilot editing interface, allowed pilots to enter into altitude, speed, and heading holds without modifying their mission route. Heading holds could be executed through numerical inputs to a steering command window or through interaction with a graphical compass rose interface (shown in Fig. 3). The compass rose interface allowed pilots to drag a heading bug to their desired direction rather than enter the value manually. Altitude and speed holds, however, could only be executed using numerical inputs to the steering command window. Pilots uploaded changes to the aircraft by pressing a "Send" button within the steering command window. As will be discussed in detail below, the TSD was also configured to display surrounding traffic, alerting information, and conflict resolution tools in select conditions. Unless otherwise stated, the TSD was restricted to the functionality described here.

A health and status panel was positioned to the right of the TSD and contained subsystem information, telemetry data, a chat client, and an electronic checklist. The third VSCS display was a simulated out-the-window nose-camera 


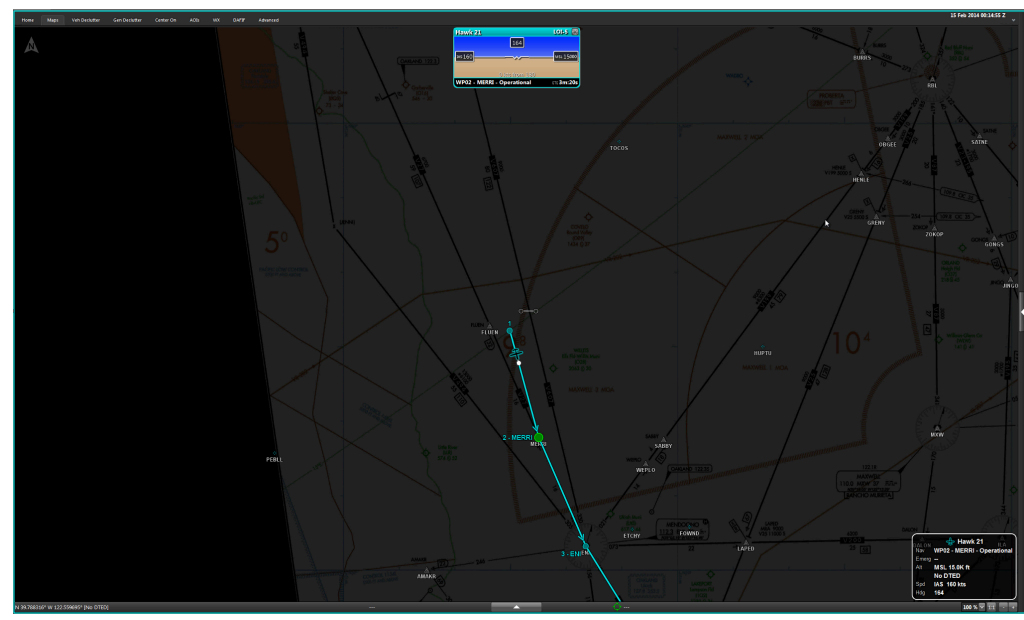

Figure 2. Vigilant Spirit Control Station tactical situation display (AFRL/RH). Distribution A: Approved for public release; distribution unlimited, 3/18/2013; 88ABW-2013-1303.

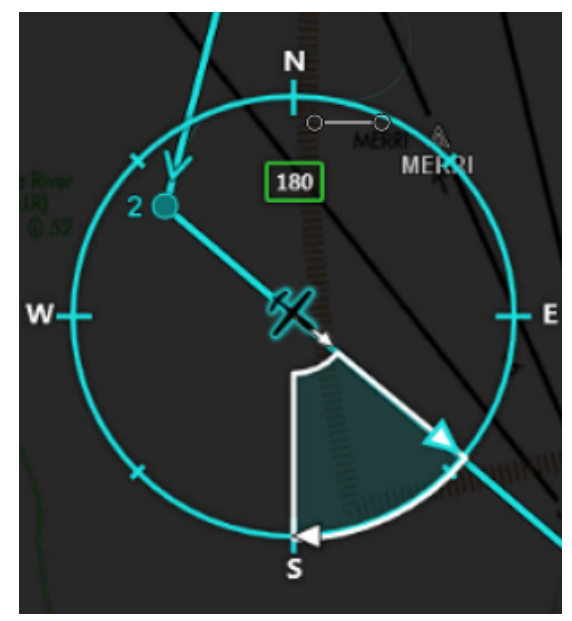

Figure 3. Vigilant Spirit Control Station's compass rose interface.

view positioned directly above the TSD. This monitor included synthetic terrain and an integrated head up display with current airspeed, altitude and heading information. All interaction with the three VSCS components occurred through standard mouse and keyboard inputs.

A fourth monitor was populated by the CSD (shown in Fig. 4), a 3D volumetric cockpit display of traffic information developed by the Flight Deck Display Research Laboratory at NASA Ames Research Center ${ }^{15}$. The CSD, positioned directly to the left of the TSD on a separate monitor, was configured to display ownship information, surrounding traffic and alerting information, and, in select conditions, conflict detection tools. Pilot interaction with the CSD, while limited, was enabled through standard keyboard and mouse inputs. Pilots were able to adjust the display range of the CSD by using the mouse's scroll wheel within the boundary of the volumetric display or by using a range dial on an external menu. The CSD had a minimum display range of $10 \mathrm{~nm}$ and a maximum display range of $640 \mathrm{~nm}$. Along with intruder and ownship information, the CSD provided pilots with range rings and heading markers along the edge of the display. For the purposes of this experiment, the CSD was

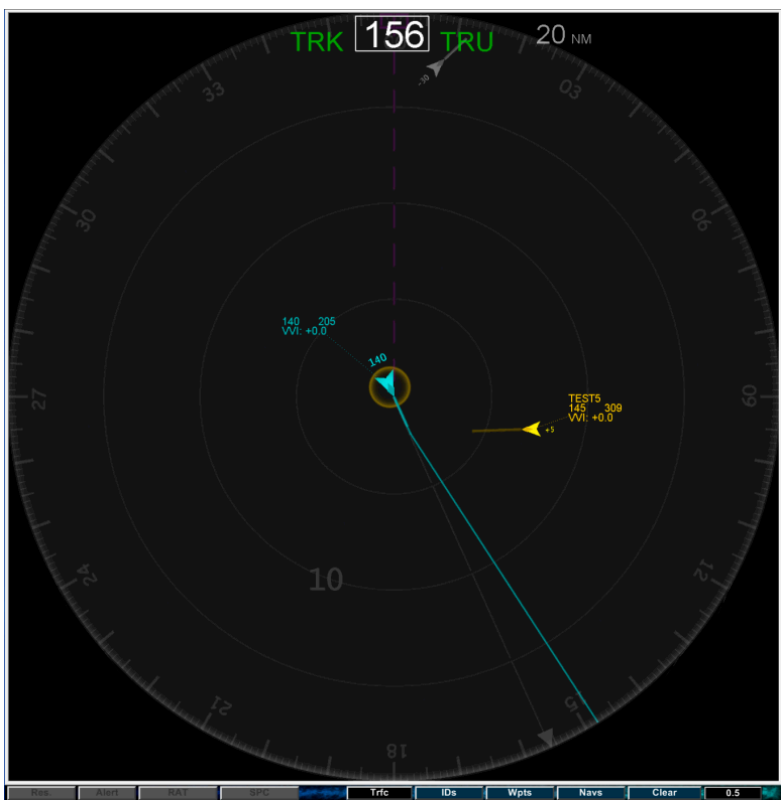

Figure 4. The Cockpit Situation Display (CSD), developed by the Flight Deck Display Research Laboratory. restricted to a 2D top-down view and an ego-centric, northup orientation.

\section{Detect and Avoid System}

The DAA system utilized in this experiment was provided by the Java Architecture for DAA Modeling and Extensibility $^{16}$. JADEM served as this study's surveillance and threat detection system. The software was configured to replicate ADS-B, with a lateral sensor range of $80 \mathrm{~nm}$ and a vertical sensor range of $+/-5000$ feet. Aircraft outside of this range were not displayed to the pilot. JADEM also contained conflict detection logic that evaluated and prioritized surrounding traffic according to their predicted threat level with ownship. To calculate the predicted threat level, JADEM compared ownship's known intent to surrounding traffic, extrapolating the intruder's future position assuming their constant velocity. The spatial and temporal thresholds used to determine threats, along with their associated threat alerting levels, are described in further detail below. Finally, JADEM also provided pilots in the advanced display conditions with text-based directive guidance through its conflict resolution algorithm, Autoresolver-AD ${ }^{16}$. Autoresolver-AD presented pilots with a recommended maneuver, in the form of a text box that 
was calculated as inducing the least amount of delay for resolving the active threat. If Autoresolver-AD was unable to generate a threat-free maneuver, the algorithm presented pilots with a maneuver that maximized the horizontal miss distance between ownship and the intruder. If at any point Autoresolver-AD computed a more effective (i.e., safer or more efficient) maneuver, pilots were given the ability to replace the previous recommendation with the latest solution. Autoresolver-AD was disabled in the basic display conditions.

3. Traffic Simulation

The Multi-Aircraft Control Station (MACS) provided the air traffic simulation environment for this study ${ }^{17}$. MACS was used to generate simulated traffic targets, the confederate controller's display, and two pseudo pilot stations. An en route ATC display provided the confederate controller with the ability to realistically manage all traffic within the designated experimental sector (Oakland Center ZOA 40/41). Two pseudo pilot stations enabled confederate pilots to take control of, and respond as, any manned aircraft in the simulated airspace. All experimental participants communicated over a single voice IP communication application.

\section{Experimental Design}

This study utilized a within-subjects, repeated measures factorial design to compare the effect of information level and display location on UAS pilots' performance on maintaining self separation and collision avoidance from other aircraft while operating in civil airspace. Two levels of information (basic, advanced) were compared across two levels of display location (standalone, integrated), thus a total of four displays were examined: 1) basic standalone, 2) basic integrated, 3) advanced standalone, and 4) advanced integrated.

1. Information Level

The results from Friedman-Berg et al. ${ }^{11}$ and Draper et al. ${ }^{12}$ discussed above, were crosschecked against existing relevant references and documents (e.g., DO-317 Minimum Operational Performance Standards for Aircraft Surveillance Applications System ${ }^{18}$ ) in order to come up with a set of minimum information requirements that could be evaluated in this experiment as the basic condition. The information set for the basic display condition is listed in Table 2 .

Table 2. The minimum information set for the basic display condition.

\begin{tabular}{|c|c|c|c|}
\hline Intruder Information & Visibility & Ownship Information & Visibility \\
\hline Location & Always visible & Location & Always visible \\
\hline Range & Always visible & & \\
\hline Bearing & Always visible & Trajectory & Always visible \\
\hline Heading & Always visible & Heading & Always visible \\
\hline Relative Altitude & Always visible & Altitude & Always visible \\
\hline Vertical Trend & Always visible & Vertical Trend & Always visible \\
\hline Heading Predictor & Always visible & & \\
\hline Alert/Threat Level & Always visible & & \\
\hline Vertical Velocity & Within data tag & Vertical Velocity & Within data tag \\
\hline Absolute Altitude & Within data tag & & \\
\hline Ground Speed & Within data tag & Ground Speed & Within data tag \\
\hline Aircraft ID & Within data tag & & \\
\hline
\end{tabular}

The visibility of each information element was also specified, that is, whether it should be always visible on the DAA display, or whether it could be shown in a data tag. Information shown within a data tag was only displayed if: 1) the data tag was selected for an intruder with a Proximal or Preventative threat level (see below), or 2) an aircraft had a self separation or collision avoidance threat level (at which point the data tag information was automatically visible).

Table 3 presents the multi-level alerting structure that was used in the basic display condition. Threat level was based on the location of and time to the closest point of approach (CPA) between the ownship and an intruder aircraft. Both the location and time thresholds had to be met for an intruder to be assigned a threat level. Location of the CPA was measured by both the lateral distance, in nautical miles (nm), and vertical distance, in feet (ft), to the 
predicted CPA location. The time to CPA was measured in seconds (sec). For this experiment, the collision avoidance and well clear thresholds were treated as equivalent.

Table 3. The multi-level alerting structure used in the basic display condition.

\begin{tabular}{lllll}
\hline Alert/Threat Level & \multicolumn{2}{l}{ CPA Distance from Ownship } & Time to CPA & Color \\
Lateral & $>2 \mathrm{~nm}$ & $>900 \mathrm{ft}$ & N/A & Grey \\
Proximal & $<2 \mathrm{~nm}$ & $<900 \mathrm{ft}$ & $<120 \mathrm{sec}$ & White \\
Preventative & $<1.2 \mathrm{~nm}$ & $<900 \mathrm{ft}$ & $<110 \mathrm{sec}$ & Yellow \\
Self Separation & $<0.8 \mathrm{~nm}$ & $<400 \mathrm{ft}$ & $<40 \mathrm{sec}$ & Red \\
Collision Avoidance & & & \\
\hline
\end{tabular}

The advanced display condition contained all of the information elements within the basic display condition, but added additional features to assist the pilot in maintaining self separation and collision avoidance from other aircraft. These additional features included: an additional predicted collision avoidance alerting level, a depiction of intruder and ownship predicted CPA location, a $0.8 \mathrm{~nm}$ collision avoidance or well clear threshold "ring," a vertical situation display, a single maneuver recommendation, and trial/vector planning tools. These tools are discussed further under each specific display description below.

2. Display Location

For this experiment, the standalone DAA display condition could only receive ownship state and trajectory information from the navigation system, and could not send any information to it (e.g., to the command and control interface). The integrated display condition saw the DAA display features integrated directly into the moving map display of the GCS. The DAA display was also integrated with the navigation system. Where possible, display features were integrated with the command and control interface. The four separate DAA displays are described below.

\section{Basic Standalone}

The basic standalone DAA display condition presented pilots with the minimum information set (see Table 2) within the CSD. In order to replicate a completely independent system, the standalone display was configured to receive information from the UAS navigation system but was not capable of sending any information directly to the aircraft. All command and control changes to the aircraft were required to be made through the TSD. As shown above in Fig. 4, the basic standalone display provided pilots with minimal ownship, intruder, and alerting information. No guidance or conflict resolution tools were present in this display condition.

\section{Basic Integrated}

The basic integrated DAA display condition presented pilots with the same minimum information set but within the VSCS TSD (Fig. 5). This configuration allowed pilots to view traffic within their primary display, collocated with their vehicle control interfaces. The integrated display also allowed pilots to open and/or reposition data tags anywhere within the map area. No guidance or conflict resolution tools were present in this display condition.

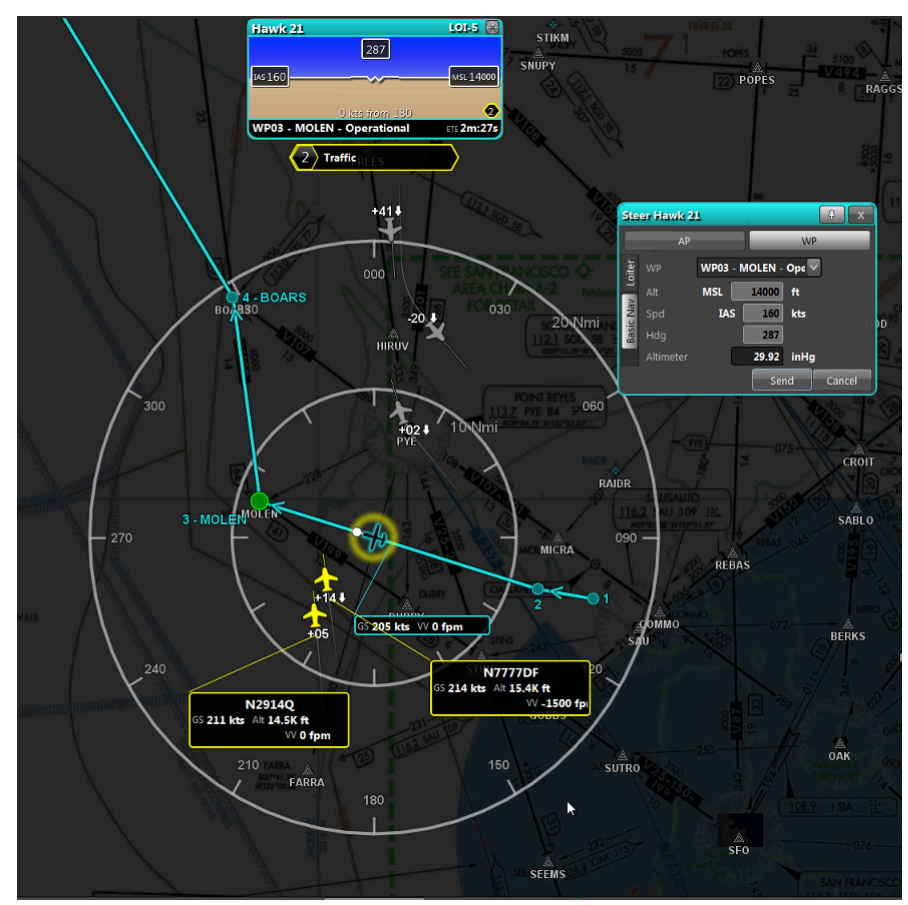

Figure 5. The basic integrated DAA display with two active self separation alerts. 


\section{Advanced Standalone}

In the advanced standalone DAA display condition (Fig. 6), pilots were provided advanced alerting information and resolution tools within the standalone display to aid in assessing and resolving conflicts. These advanced features were provided in addition to the minimum information set employed by the basic display conditions. Pilots were not required to use or follow the guidance provided by the resolution tools included in this condition.

6. Advanced Alerting Information.

CPA Location. At the onset of a self separation alert, the predicted physical location of the intruder's CPA to ownship was depicted by a color-coded circle. The CPA location remained on the display as long as a threat was active.

Time to CPA. A countdown timer was triggered by the onset of a self separation alert, indicating the time remaining until CPA was reached. The time was displayed in the bottom left-hand corner of the display and disappeared as soon as an alert was cleared.

Predicted Severity. An additional predicted collision avoidance alert level was introduced to show severity (shown in Table 4). The predicted collision avoidance alert level was intended to aid pilots in discriminating between critical and noncritical self separation threats. The existence of larger spatial thresholds for self separation alerts compared to collision avoidance alerts meant that some self separation alerts never progressed to

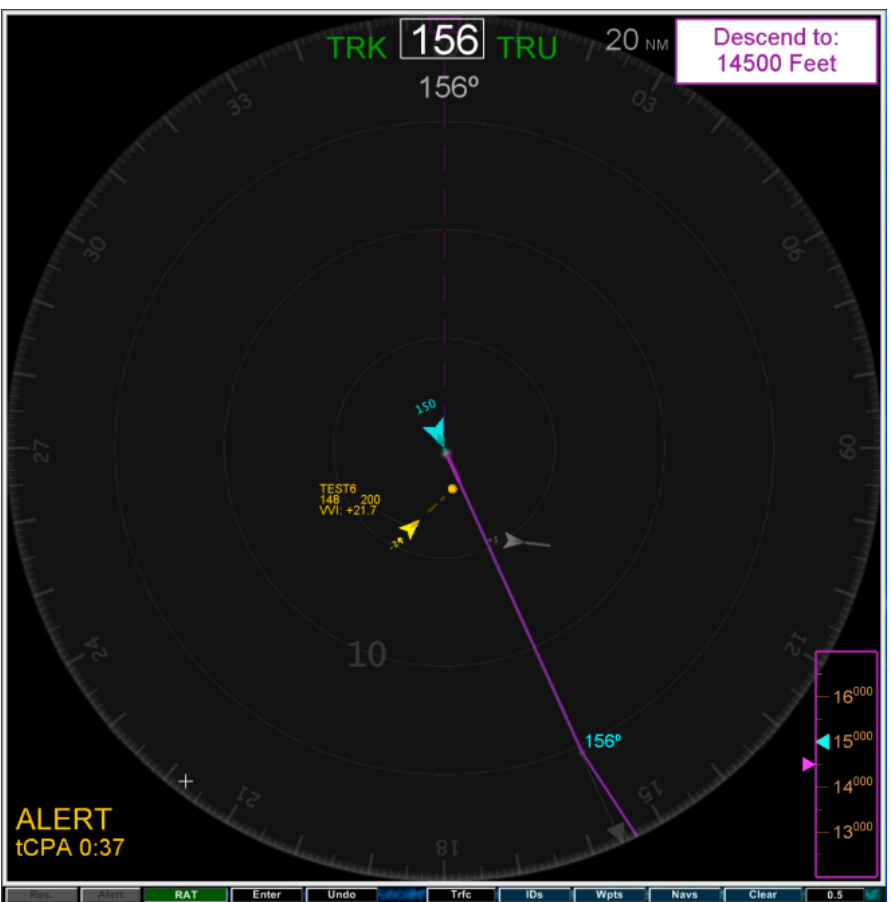

Figure 6. Screenshot of the advanced standalone DAA display condition during a self separation alert. The maneuver recommended by Autoresolver-AD is shown in the upper right-hand box ("Descend to: 14500 Feet"), the lateral trial planner is indicated by the magenta colored flight plan, the vertical trial planner (i.e., altitude tape) is located on the lower right side of the display, and the time to the predicted closest point of approach with the self separation alert is located in the bottom left.

collision avoidance alerts. The predicted collision avoidance alert level indicated critical self separation alerts (that were predicted to progress to a collision avoidance alert) by coloring the border of the CPA circle red. For less critical self separation alerts (i.e., those not predicted to become collision avoidance alerts), the CPA circle remained solid yellow.

Table 4. The multi-level alerting structure used in the advanced display conditions.

\begin{tabular}{|c|c|c|c|c|}
\hline \multirow{2}{*}{ Alert/Threat Level } & \multicolumn{2}{|c|}{ CPA Distance from Ownship } & \multirow[b]{2}{*}{ Time to CPA } & \multirow[b]{2}{*}{ Color } \\
\hline & Lateral & Vertical & & \\
\hline Proximal & $>2 \mathrm{NM}$ & $>900 \mathrm{FT}$ & N/A & Grey \\
\hline Preventative & $<2 \mathrm{NM}$ & $<900 \mathrm{FT}$ & $<120 \mathrm{SEC}$ & White \\
\hline Self Separation & $<1.2 \mathrm{NM}$ & $<900 \mathrm{FT}$ & $<110 \mathrm{SEC}$ & Yellow \\
\hline Predicted Collision Avoidance & $<0.8 \mathrm{NM}$ & $<400 \mathrm{FT}$ & $<110 \mathrm{SEC}$ & Yellow, Red Border \\
\hline Collision Avoidance & $<0.8 \mathrm{NM}$ & $<400 \mathrm{FT}$ & $<40 \mathrm{SEC}$ & Red \\
\hline
\end{tabular}

\section{Advanced Resolution Tools}

Recommended Maneuvers. At the onset of a self separation alert, Autoresolver-AD provided pilots with a recommended resolution maneuver. The maneuver appeared in a text box in the upper right hand of the display. If Autoresolver-AD computed a more effective maneuver, a "Refresh" button flashed continuously at the bottom of the display. When pressed, the new resolution maneuver replaced the previous one.

Trial Planning Tools. Two separate trial planning tools, each intended to give pilots the ability to 'test' various heading and altitude vectors by providing immediate feedback as to the quality of a proposed maneuver, were

9

American Institute of Aeronautics and Astronautics 
included. The tools were engaged automatically during self separation alerts and populated with the maneuver recommended by Autoresolver-AD. However, pilots were also able to engage the trial planning tools manually by selecting a dedicated button ("RAT") at the bottom of the display.

The lateral trial planning tool allowed pilots to superimpose a 'proposed' route line on top of their active route. The proposed route line could be manipulated without any impact to their active route. Using the superimposed route line, pilots could trial plan different heading vectors by clicking and dragging on a waypoint off the nose of the aircraft. As pilots moved a proposed waypoint away from their active route, a heading readout appeared adjacent to the waypoint, informing pilots of the exact heading vector being trial planned. The proposed route line was colorcoded based on the predicted alert level for the trial-planned heading. Proposed headings that were predicted to lead to only proximal alerts turned the line magenta, while headings predicted to lead to at least one preventative alert turned the line white. Magenta was used instead of the corresponding grey color for proximal alerts, in order to be more visually salient to the pilot and distinctive from the actual current trajectory. Proposed headings that were predicted to lead to at least one self separation alert or at least one collision avoidance alert turned the route line yellow or red, respectively. While trial planning, halos appeared around intruders that were predicted to become a proximal, self separation or collision avoidance alerts. The halos were color-coded according to the predicted alert level of the associated intruder.

Similar to the lateral tool, the vertical trial planning tool allowed pilots to probe different altitudes for their relative safety. In order to trial plan different altitudes, pilots manipulated an altitude tape positioned in the bottom right-hand corner of the display. The altitude tape included three different altitude 'bugs': a current altitude bug, a commanded altitude bug, and a trial plan altitude bug. The altitude tape was centered on the trial plan altitude bug, allowing pilots to test various altitudes by clicking and dragging the altitude tape's surface. The trial plan altitude bug and the border of the altitude tape were then color coded using the same alerting logic described for the lateral trial planning tool.

\section{Advanced Integrated}

In the advanced integrated DAA display condition, pilots were provided advanced alerting information and resolution tools within the VSCS TSD to aid in assessing and resolving conflicts (Fig. 7). These advanced features were provided in addition to the basic information and alerting set employed by the basic display conditions. As with the advanced standalone display condition, pilots were not required to use or comply with the alerting and guidance information.

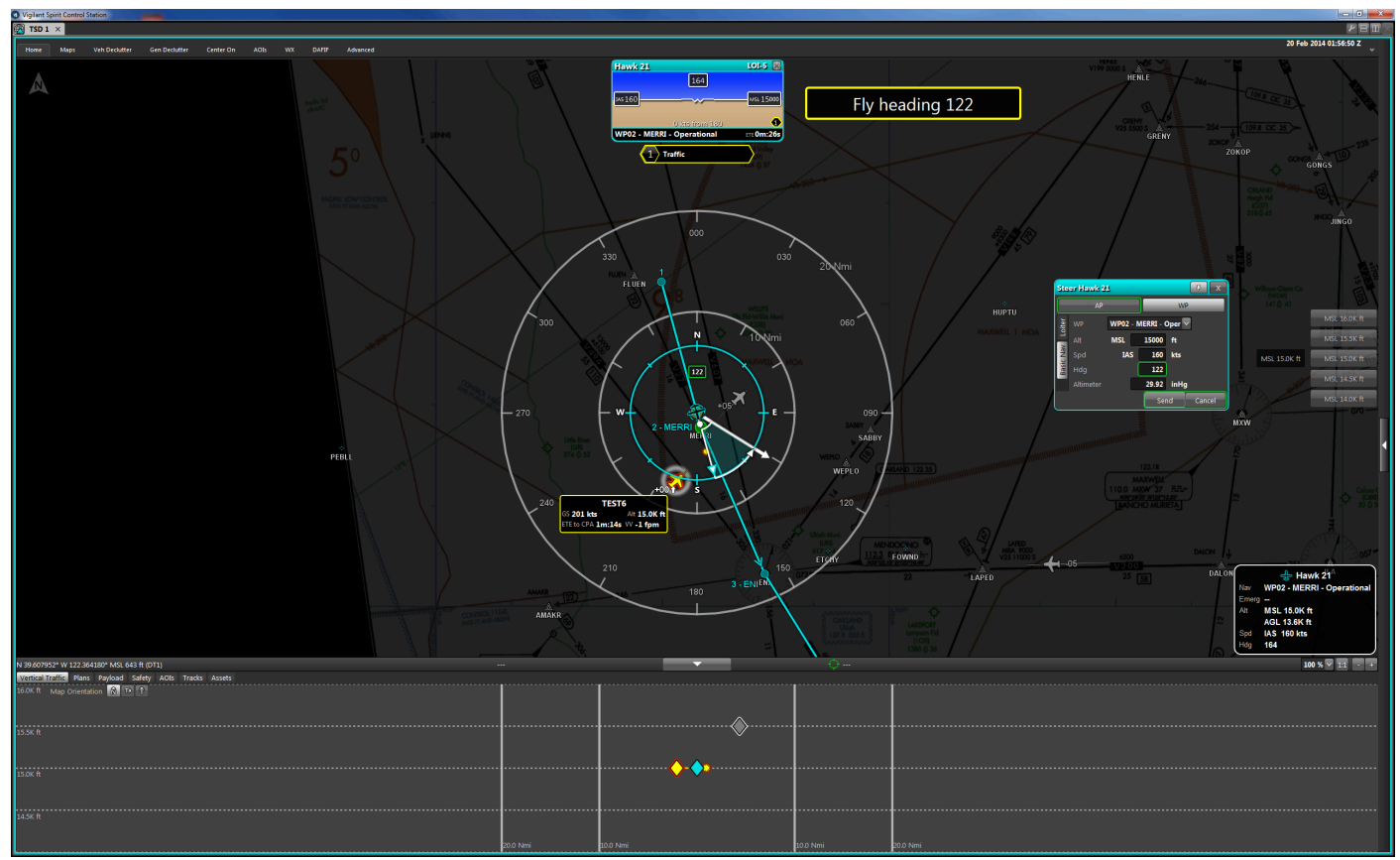

Figure 7. Screenshot of the advanced integrated DAA display condition with an active predicted collision avoidance threat. The maneuver recommended by Autoresolver-AD is shown in the upper box ("Fly Heading 122"), the lateral trial planner is indicated by the arrow pointing to heading 122 off the nose of the ownship icon (center), the vertical trial planner is located on the far right side of the TSD, and the vertical situation display is shown in the lower quarter of the display. 


\section{Alerting Information}

CPA Location. The predicted physical location of a self separation threat's CPA to ownship was presented using a color-coded circle on the display. This condition also allowed pilots to display ownship's CPA to the intruder aircraft by hovering over the threat's aircraft icon. The CPA locations automatically disappeared once an alert was cleared.

Time to CPA. A countdown timer to CPA was triggered by the onset of a self separation alert. The timer was displayed in the data tag of the relevant aircraft. This data block field was empty when the target was not an active threat to ownship.

Predicted Severity. The additional predictive collision avoidance alerting level was employed in this display condition as well (see Table 4). Whereas a red border on the CPA circle in the advanced standalone condition indicated the predicted severity, critical self separation alerts in the advanced integrated condition were indicated using a red border around both the CPA circle and the threat's aircraft icon.

Well Clear Ring: The presence of a self separation alert enabled the appearance of a "well clear ring" around ownship. With a radius of $0.8 \mathrm{~nm}$, the ring gave pilots a visual reference to the lateral collision avoidance threshold.

Vertical Situation Display. A panel at the bottom of the TSD displayed a vertical profile of traffic $+/-1,000$ feet vertically from ownship. The Vertical Situation Display (VSD) showed traffic icons, heading predictors, CPA location, and appropriate color-coding for alert level. Range rings within the VSD were designed to align with the range rings on the TSD.

\section{Advanced Resolution Tools}

Recommended Maneuvers. At the onset of a self separation alert, Autoresolver-AD provided pilots with a recommended maneuver. The text-based recommendation was displayed in the upper-right hand corner of the TSD. If Autoresolver-AD computed a more effective maneuver, a "Refresh" button appeared directly below the recommended maneuver text. When pressed, the new resolution maneuver replaced the previous one.

Trial Planning Tools. As with the advanced standalone condition, two separate trial planning tools, lateral and vertical, were included in the display. While the tools' overall function was still to allow pilots to test various heading and altitude vectors, their implementation had to be modified to allow for integration with the vehicle controls. Once again, the trial planning tools were automatically engaged at the onset of a self separation alert and populated with the maneuver recommended by Autoresolver-AD. The tools could also be launched manually in the absence of an active self separation alert.

The lateral trial planning tool was integrated into the TSD's autopilot interface. At the onset of a self separation alert the compass rose automatically opened on the TSD and a vector arrow appeared, extending from ownship to the edge of the compass rose in the direction of the recommended Autoresolver-AD maneuver. As pilots dragged the heading bug, the collocated arrow gave instantaneous feedback as to the quality of the proposed heading. As with the proposed route line in the advanced standalone condition, the vector arrow was color-coded based on the safety level of the heading being probed. Proposed headings that were predicted to lead to only proximal alerts turned the vector arrow green, while headings predicted to lead to at least one preventative alert turned the arrow white. Proposed headings that were predicted to lead to at least one self separation alert or at least one collision avoidance alert turned the vector arrow yellow or red, respectively. Since the vector arrow was integrated into the autopilot interface, pilots could directly send any proposed heading holds up to the aircraft by pressing "Send" in the autopilot's steering command window.

The vertical trial planning tool utilized an altitude table permanently displayed on the far right side of the TSD. The altitude table consisted of five discrete altitude options: one at the current altitude, two 1,000 feet above the current altitude (in 500 foot increments), and two 1,000 feet below the current altitude (also in 500 foot increments). The same color-coding scheme as described for the lateral trial planning tool was used for the altitude table to indicate the predicted safety level of each altitude option. Each altitude option was selectable and tied to the TSD's autopilot interface. When pressed, the selected altitude was pushed to the steering command window. Pilots could then upload the new altitude to the aircraft by pressing "Send" within the steering command window. While trial planning with the lateral or vertical tools, halos appeared around intruders that were predicted to become a proximal, self separation or collision avoidance alerts. The halos were color-coded according to predicted alert level.

\section{Pilot Task}

Pilots were tasked with operating a simulated MQ-9 Reaper, "HAWK21," along one of two pre-filed flight paths within Oakland Center airspace (ZOA 40/41). Pilots flew under instrument flight rules (IFR) and were responsible for navigating the aircraft and responding to a variety of scripted health and status tasks. These secondary tasks included responding to requests for status information (e.g., current fuel level) in a chat client and completing electronic checklists in response to aircraft system malfunctions. Pilots were also instructed to monitor their traffic 
display for potential safety of flight concerns. If a safety of flight threat was discovered, they were instructed to notify air traffic control of their desire to maneuver (time permitting) and to upload the change to the aircraft. They were encouraged to minimize their deviation from the flight plan and to coordinate a return to their mission route and/or altitude with air traffic control as soon as practical.

\section{Scenarios}

Pilots flew two different mission routes, a "Fire Line" mission and a "Coastal Watch" mission. Each route started with HAWK21 already at mission altitude, flying towards its second programmed waypoint. There were no scripted altitude changes for either of the mission routes. The Fire Line mission route was level at 12,000 feet and Coastal Watch mission route remained level at 14,000 feet. Two different manned traffic scenarios were scripted to run alongside the two mission routes. Both traffic scenarios were developed by an ATC subject matter expert and designed to provide equivalent pilot workload. In each scenario, eight intruders were scripted to progress to a self separation then collision avoidance alert, absent of pilot action, while four different intruders were scripted to progress only to a preventive alert. All encounters were built for a single intruder, however, dynamic changes to the surrounding traffic made it possible for multiple intruders to occur simultaneously. Live traffic data was referenced in order to help simulate traffic patterns and densities that were representative of a busy day at Oakland Center.

\section{Procedure}

\section{Training}

Participants first completed an informed consent form and a demographics form, which elicited information regarding their experience in manned and unmanned aviation. This was followed by a brief overview of the day's schedule and an introduction to the pilot tasks. Pilots then underwent extensive training on the basic functionality of VSCS. This included practice on how to use the TSD's vehicle control interfaces as well as how to perform the various health and status tasks that would be present during the experimental trials. Pilots concluded this portion of the training with a 20-minute practice scenario. Pilots received hands-on training and completed additional 20minute practice scenarios prior to all subsequent display conditions.

\section{Experimental Trials}

Participants completed four, 37-minute experimental trials. All participants received the four different DAA display conditions described above: basic standalone, basic integrated, advanced standalone, and advanced integrated. The presentation of the display conditions was counterbalanced across participants to account for order and learning effects. Following each experimental trial, participants completed the NASA Task Load Index (TLX) ${ }^{19}$ and a post-trial subjective questionnaire, which focused on the unique display elements of the preceding condition. A post-simulation questionnaire and debrief followed the final experimental trial.

\section{MEASURES}

Pilots' measured response for the DAA task was modified from the pilot-ATC interaction measured response timelines utilized in previous studies ${ }^{5,6,7}$. The pilot-DAA interaction measured response timeline was extracted from time stamps at eight discrete and operationally relevant stages of a pilot's response to a self separation (yellow and yellow with red border) or collision avoidance threat alert (red). A response encounter was defined as an interaction with a single intruder, starting from the appearance of a self separation or collision avoidance alert until these alert levels were removed and the intruder returned to a preventative or proximal alert level (see Table 3 for the multilevel alerting stages). A single alert encounter could have multiple self separation and collision avoidance alerts associated with it, however, it was only considered a "new" encounter if the self separation or collision avoidance alert was removed and then appeared again (i.e., due to changing trajectories of one or both aircraft). If a pilot completed any of the stages in the pilot-DAA timeline after the appearance of a self separation or collision avoidance alert, the measures for that response was logged.

Table 5 describes each of the eight stages of the pilot-DAA interaction timeline. (For the purposes of this study, inputs to the command and control interface in order to input a resolution maneuver are termed "edits.") The time stamps for each stage were collected from a variety of data sources including raw MACS, VSCS, and JADEM output files, voice recordings and logs, and screen recordings of the pilot display. Where possible, data sources were crosschecked with each other, and screen recordings were referenced to provide context of the results. 
Table 5. Stages of the pilot-DAA interaction timeline.

\begin{tabular}{ll}
\hline Stage & Description \\
$\mathbf{T}_{\mathbf{0}}$ & DAA (self separation or collision avoidance) alert appears on the display \\
$\mathbf{T}_{\mathbf{1}}$ & Pilot notifies ATC and requests a maneuver clearance \\
$\mathbf{T}_{\mathbf{2}}$ & ATC provides maneuver clearance \\
$\mathbf{T}_{\mathbf{3}}$ & Pilot initiates an edit in GCS to maneuver \\
$\mathbf{T}_{\mathbf{4 a}}$ & Pilot uploads $1^{\text {st }}$ maneuver to aircraft \\
$\mathbf{T}_{\mathbf{4 b}}$ & Pilot uploads final maneuver to aircraft \\
$\mathbf{T}_{\mathbf{5}}$ & Traffic alert is removed from display \\
$\mathbf{T}_{\mathbf{6}}$ & UAS completes maneuver \\
\hline
\end{tabular}

A number of pilot response time metrics were calculated based on the time-stamped stages collected in Table 5 . The metrics reported here focus on pilots' interaction with the DAA system, including the traffic displays (detection of threats and determination of a resolution) and the control and navigation interface (execution of a resolution maneuver); these metrics are most critical to understanding the effect of display configurations on pilot performance. All response times were calculated in seconds (s). Figure 8 depicts the temporal relationship between reported metrics.

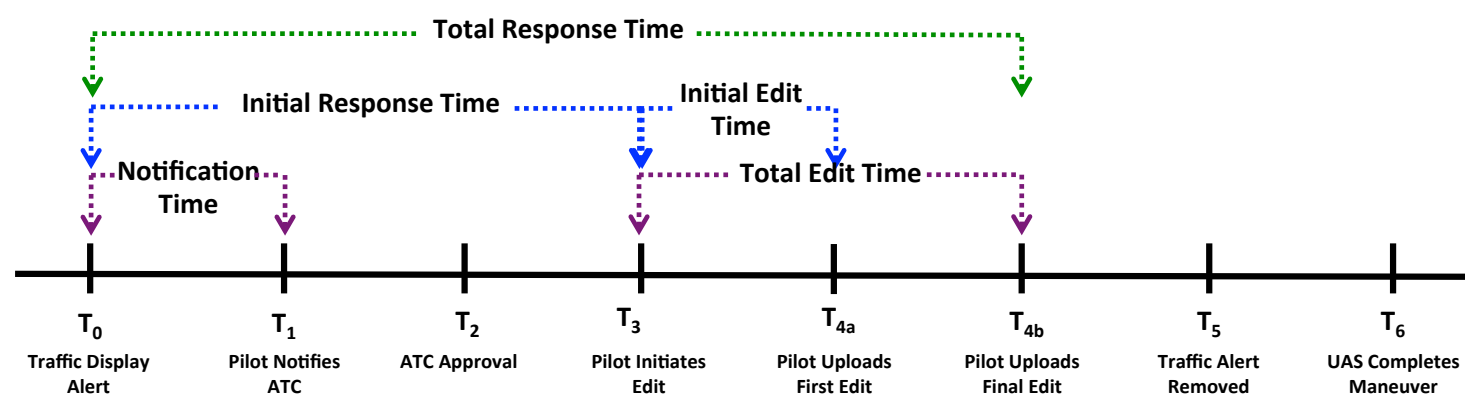

Figure 8. Temporal relationship of the pilot measured response stages and response time metrics.

Notification Time $\left(T_{1}-T_{0}\right)$. A measure of the time it takes a pilot to notify ATC of the appearance of a self separation or collision avoidance alert and the need to execute a maneuver. Calculated as the difference between the first appearance of the alert and the beginning of the pilot's transmission on the radio to notify the controller.

Initial Response Time $\left(T_{3}-T_{0}\right)$. A measure of the time it takes a pilot to initiate a maneuver response, or edit, in the GCS command and control interface in response to a traffic display alert. Calculated as the difference between the first appearance of the alert and the start of an edit.

Initial Edit Time $\left(T_{4 a}-T_{3}\right)$. A measure of the time it takes a pilot to input an initial edit into the GCS in order to maneuver in response to a traffic alert. Calculated as the time between initiating an edit and the first upload to the aircraft. This metric is only relevant if a pilot uploaded multiple edits to the aircraft; when pilots made only one edit, initial edit time is equivalent to total edit time.

Total Edit Time $\left(T_{4 b}-T_{3}\right)$. A measure of the time it takes a pilot to complete an edit into the GCS in order to maneuver in response to a traffic alert. Calculated as the time between initiating an edit and the final upload to the aircraft. The final upload is assumed to be the pilot's final resolution decision.

Total Response Time $\left(T_{4 b}-T_{0}\right)$. A measure of the time it takes a pilot to upload a final maneuver resolution to the aircraft in response to a traffic alert. Calculated as the time between the initial appearance of the traffic alert and the final maneuver upload to the aircraft.

\section{RESULTS}

Each of the five metrics listed above were analyzed utilizing a 2 (information level: basic, advanced) X 2 (display location: standalone, integrated) repeated measures Analysis of Variance (ANOVA) with Bonferroni pairwise corrections for the main effect post hoc comparisons. An alpha level of .05 was used for all analyses. Significant interactions were analyzed using $\mathrm{T}$ test comparisons. Pilots responded to a total of 261 discrete alerts, 251 alerts appeared initially at the self separation threat level, the remaining ten alerts appeared initially at the collision avoidance threat level. 


\section{A. Notification Time}

The main effect of information level on pilot notification time approached statistical significance, $F(1,11)=$ 4.432, $p=.059$. The advanced displays $(M=25.99 ; S E=2.78)$ appeared to support faster pilot notification times than the basic displays $(M=32.16 ; S E=4.85)$. There was no significant main effect of display location, $F(1,11)=$ $.140, p>.05$, nor was there a significant interaction, $F(1,11)=.018, p>.05$.

\section{B. Initial Response Time}

There was not a main effect of information level on initial response time, $F(1,11)=.459, p>.05$. However, the main effect of display location on initial response time approached significance, $F(1,11)=4.635, p=.054$. Pilots' initial response time appeared faster for the standalone displays $(M=16.78 ; S E=1.80)$ compared to the integrated displays $(M=21.85 ; S E=2.20)$. The interaction between information level and display location was not significant, $F(1,11)=.239, p>.05$.

\section{Initial Edit Time}

There was a significant interaction of information level and display location on pilots' initial edit time, $F(1,11)$ $=13.851, p<.01$. Post hoc pairwise $\mathrm{T}$ tests revealed a significant difference in initial edit time between the basic $(M=17.11 ; S E=3.11)$ and advanced $(M=5.51 ; S E=3.42)$ displays for the integrated display condition, $t(11)=$ $3.449, p<.01$. However, the difference between basic and advanced was not significant for the standalone condition displays, $t(11)=1.126, p>.05$. The difference between basic and advanced for the integrated displays was large enough to result in a significant main effect of information level on initial edit time, $F(1,11)=8.972, p<$ .05 . There was not a significant main effect of display location on initial edit time, $F(1,11)=.139, p>.05$.

\section{Total Edit Time}

Information level had a significant effect on total edit time, $F(1,11)=11.821, p<.01$ (Fig. 9). The advanced displays $(M$ $=13.18 ; S E=2,48)$ had significantly shorter edit times than the basic displays $(M=22.12 ; S E=3.14)$. There was not a significant main effect of display location on total edit time, $F(1,11)=1.192, p>.05$, nor was there a significant interaction between information level and display location, $F(1,11)=2.804, p>.05$.

\section{E. Total Response Time}

There was a significant main effect of information level on total response time, $F(1,11)=6.619, p<.05$ (Fig. 10). On average, the advanced display condition $(M=33.98 ; S E=$ $3.34)$ was $13.79 \mathrm{~s}$ faster than the basic display condition $(M=$ 41.77; $S E=3.53$ ). The main effect for display location and the interaction were both non-significant, $F(1,11)=.633$ and $F(1,11)=2.472$, respectively, $p>.05$.

\section{DISCUSSION}

The primary purpose of a DAA display for UAS is to enable pilots to carry out three primary DAA functions: detect potential threats, determine appropriate resolution maneuvers, and execute those maneuvers via the GCS control and navigation interface. A number of discrete stages mark key pilot activities along the pilot-DAA timeline (Table 5). The eight stages map roughly, though not perfectly, to the three DAA functions a pilot is responsible for. The execute function has the most clear mapping to the final upload $\left(\mathrm{T}_{4 \mathrm{~b}}\right)$. The detection function can be measured as some time after the first alert, $\mathrm{T}_{0}$, and before the next stage that the pilot

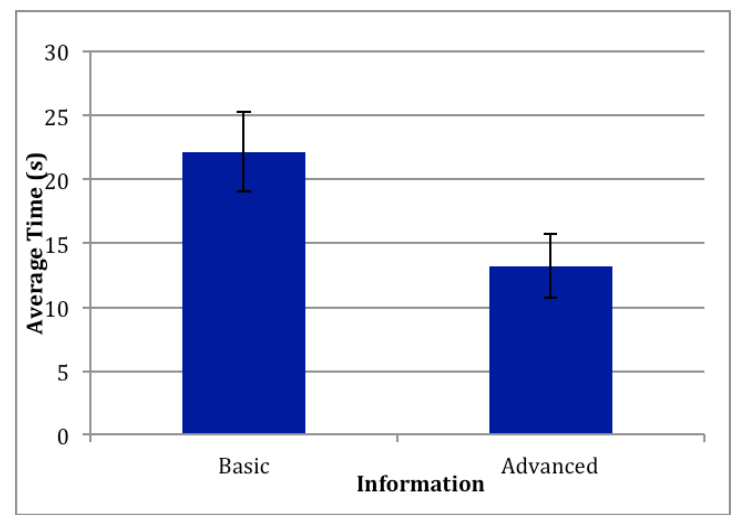

Figure 9. Total edit time by information level.

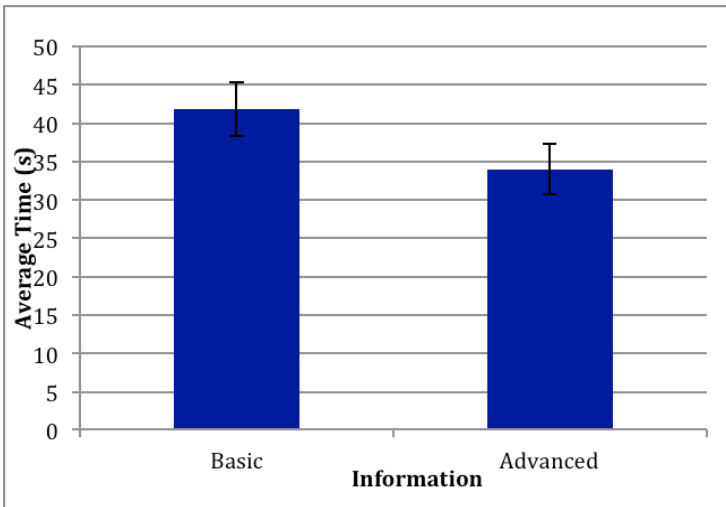

Figure 10. Total response time by information level. executes, for example, notifying ATC of the need to maneuver $\left(T_{1}\right)$ or initiating a maneuver response in the GCS ( $\mathrm{T}_{3}$; pilots did not always request a clearance prior to maneuvering). The determine function is the most difficult to 
map, pilots can be actively determining an appropriate maneuver any time between when the alert appears and the final maneuver is executed, essentially the entire timeline that the pilot is engaged in the DAA task. Despite the challenge of directly mapping the eight timeline stages to the pilot's DAA functions, a number of pilot performance metrics can be extracted and provide insight to pilots' activities during these stages. The above results show that these metrics are sensitive to various display configurations, especially differences in information across configurations.

As hypothesized, advanced information displays showed an advantage in terms of pilot performance over the basic, or minimum information, displays. Both the standalone and integrated versions of the advanced display condition showed significantly faster response times than the basic display conditions for two of the reported metrics: total edit time and total response time. In addition, the integrated advanced display supported significantly faster initial edit times than the integrated basic display. Surprisingly however, there were no significant findings for display location; of the six metrics reported only one, initial response time, showed a near significant difference between standalone and integrated. The implication of each metric in the pilot-DAA interaction timeline, along with the results for each metric in the present study, is discussed below.

\section{A. Notification Time}

Notification time is a measure of the time it takes for a pilot to notify ATC of a potential conflict and the need to execute a resolution maneuver. A pilot could notify the controller and request a maneuver prior to maneuvering, or notify the controller after maneuvering. In this study, pilots took an average of 29 seconds to notify ATC of a potential conflict. Interestingly, the average notification time was longer than the average initial response time of 19 seconds, thus pilots typically began initiating a maneuver prior to notifying ATC. This could be because pilots wanted to determine an appropriate maneuver before requesting a clearance, or it could be because pilots were too busy determining a maneuver to notify ATC. Overall, pilots notified ATC of a potential conflict and subsequent maneuver $97 \%$ percent of the time. However, this notification occurred prior to maneuver execution only $65 \%$ of the time, which indicates that pilots sometimes felt that a potential conflict was too urgent to allow time to request a maneuver clearance.

There were no significant differences in notification time between the four display conditions, although the difference between the advanced and basic display approached significance. The slightly faster notification times may reflect that the pilots were faster at the entire DAA task (i.e., total response time) when advanced information and tools were present in the DAA display.

\section{B. Initial Response Time}

Initial response time is a measure of the time it takes for a pilot to initiate a maneuver in the GCS control and navigation interface after the appearance of an alert on the DAA display. This time includes the time it takes the pilot to detect a potential threat. In addition, it may include some time where the pilot is determining an appropriate resolution maneuver. Differences in initial response time could be due to differences in the time for pilots to detect a potential threat, differences in the amount of time spent determining a resolution maneuver, or differences in control and navigation interfaces (see Rorie \& $\mathrm{Fern}^{7}$ for a discussion). Since this study utilized the same control and navigation interface for all four display conditions, potential differences would likely be a result of variation in the DAA displays.

There was not a significant difference between the advanced and basic display conditions, which suggests that information differences did not affect initial response time. However, there was a near significant difference between the standalone and integrated displays, whereby the standalone condition was nearly 5 seconds faster. A potential difference here could be explained by pilots initiating a maneuver sooner in the standalone condition in anticipation of a potential threat (before it became a self separation or collision avoidance alert on the display) in order to compensate for the separate displays. In fact, the distribution of initial response times indicates that pilots initiated a maneuver prior to a self separation or collision avoidance alert for $7 \%$ of all maneuvers.

\section{Initial and Total Edit Times}

Initial and total edit times reflect the amount of time it takes for a pilot to input and upload a first and final maneuver to the aircraft, respectively, from the initiation of that input. In other words, it is the amount of time that pilots interact directly with the GCS. Edit times capture most (if not all) of the time spent determining an appropriate resolution maneuver; although pilots likely begin to think about a maneuver prior to initiating a response, the lack of significant differences in initial response time compared to large significant differences in edit times between the basic and advanced information conditions, suggests that pilots execute much of the determine function within the edit time. Initial and final upload times are captured because pilots sometimes make multiple uploads to the aircraft, 
using subsequent uploads to tweak or correct their initial upload. Pilots operating with the advanced displays were, on average, 9 seconds faster at inputting and uploading their final resolution maneuvers, although the difference between the advanced and basic information display conditions was only significant for the integrated displays at the first upload, (5.5s and $17.1 \mathrm{~s}$, respectively - a $68 \%$ improvement for the advanced display).

The advanced displays were on average 9 seconds, or $40 \%$ faster than the basic displays on total edit time. Thus, it appears that the additional information and tools provided by the advanced display conditions assisted pilots in more quickly determining and uploading a maneuver in response to displayed self separation and collision avoidance alerts. Although the authors hypothesized that the response times overall would also be faster for the integrated displays compared to the standalone displays, especially for the advanced integrated display where the advanced tools were integrated with the GCS's control and navigation interface, there were no significant differences in either initial or total edit between the standalone and integrated displays.

Advantages in edit times, do not necessarily equate to overall faster response times, as it only reflects one stage of a pilot's functions to determine and then execute a resolution maneuver. Edit times only capture the time that pilots interact directly with the GCS, and not the time prior to interacting with the GCS which may be spent just visually examining the DAA display. A slower response earlier in the timeline could negate any benefits found in the edit times for a particular display configuration. Total response time captures initial response time in addition to total edit time, respectively.

\section{Total Response Time}

Total response time captures the time that it takes for pilots to upload their final maneuver in response to a display alert, from when the alert first appears. Total response time captures all three pilot DAA functions and the entire pilot-in-the-loop activity of the DAA system. This is the pilot performance metric that DAA system designers and standards developers are most concerned with, and the biggest unknown in the complete system. Total response time represents the time from the DAA alert appearing until the pilot completes their portion of the DAA timeline and the aircraft executes the final, commanded maneuver. Pilots completed their DAA functions about 14 seconds, or $33 \%$ faster with the advanced information displays than with the basic information displays. A 14 second difference in total response time could have a significant impact on the potential for a well clear violation, depending on the rate of closure between the ownship and another aircraft. As mentioned previously, pilots will potentially face constrained timeframes for responding. For this experiment, the well clear threshold was set at 40 seconds to closest point of approach and the alerting threshold at 110 seconds, leaving 70 seconds for the pilot and aircraft to respond and maneuver in time to avoid a potential threshold violation. There are essentially two buffers on each side of a pilot's total response time prior to a well clear violation: 1) a buffer for when a potential threat is detected (i.e., some allowance for a later detection due to sensor performance or encounter geometry), and 2) a buffer for aircraft performance (and lower restrictions on aircraft performance reduce costs on manufacturers and allow a wider variety of aircraft to meet defined standards and regulations). Shorter total response times for a pilot to respond to a DAA alert could allot for greater buffers for late detections and more limited aircraft performance.

\section{E. Summary}

Overall, the metrics extracted from the proposed eight stages of pilot-DAA interaction appear to be useful in quantifying and understanding the pilot DAA task functions of detecting potential threats, determining a resolution maneuver, and executing that maneuver via the GCS command and control interface. In this study, these metrics were sensitive primarily to differences in the amount and kind of information between the advanced and basic display conditions. The advanced displays resulted in faster response times than the basic displays for three of the five reported metrics. Most importantly, the advanced information displays showed significantly faster total response times than the basic information displays, a metric that captures the entire pilot DAA task, from the presentation of a self separation or collision avoidance alert to execution of the final resolution maneuver. This finding could have important implications for the development of minimum information requirements for DAA displays. The basic information condition in this study represented a first attempt to establish a minimum information set, however, it's poorer performance in terms of slower pilot response times compared to the advanced information displays could indicate that the information presented to pilots was not sufficient. As noted earlier, delayed pilot response times increase the potential of violating separation and collision avoidance thresholds.

It is important to note here that the results of this study alone do not yet support any final conclusions. First, the results of this study need to be assessed in conjunction with the loss of separation or well clear results reported by Santiago \& Mueller ${ }^{4}$. Although faster pilot response times are considered more desirable, this is only true if the faster response times coincide with fewer losses of well clear; if for some reason pilots are faster but cause more 
losses of well clear, the information provided in the display needs to be reassessed. In addition to examing the loss of well clear results, a safety analysis is required to determine whether the observed level of pilot performance (both in terms of response times and well clear violation rates) meet a defined airspace safety threshold. Second, although the results suggest that the minimum information set from this study may not be sufficient, it does not automatically lead to the conclusion that the full advanced information suite is a necessary requirement for DAA displays. It is possible that only one or two features of the advanced displays contribute to improved pilot performance and are required in the minimum information set. Finally, the lack of significant differences between the standalone and integrated condition, despite the hypothesis that the integrated displays would support faster response times overall, also begs additional investigation. Before accepting the null hypothesis, that there was no performance impact between the two display configurations, a number of additional operational conditions need to be examined. For example, when the DAA display and the GCS tactical situation display or moving map are at different zoom levels or different orientations. Or when the DAA display is in different locations within the GCS. Clearly, further work is needed to identify what constitutes the minimum display requirements that will satisfy the airspace safety threshold.

\section{CONCLUSION}

The simulation experiment described above presents a productive first step in the vast amount of work needed by the UAS community to quantify the minimum operational performance standards for a UAS DAA system. Specifically, this study quantified the pilot response time on the DAA task in order to contribute to defining the minimum information that a pilot needs presented on a DAA display to maintain self separation and collision avoidance from other aircraft. The first of several planned studies, the development of the simulation capabilities and initial display concepts, including the algorithms underlying the advanced information tools, facilitate a number of follow on studies and fruitful research opportunities to support continued investigation of issues critical to the integration of UAS into the NAS. Future studies will need to further the work presented here by examining the effect on pilot performance of some of the individual advanced information features tested in this study, different surveillance sensor parameters and performance, alternative ways of display resolution information and guidance, alternative DAA algorithms, alternative GCS configurations (including different control and navigation interfaces), communication delays, automation, and more.

\section{References}

${ }^{1}$ Federal Aviation Administration. "Integration of civil unmanned aircraft systems (UAS) in the national airspace system (NAS) roadmap, first edition." FAA, Washington, D.C, 2013.

${ }^{2}$ RTCA. "Terms of reference RTCA special committee 228 minimum performance standards for unmanned aircraft systems,” paper No. 109-13/PMC-1089. RTCA Inc., Washington, DC, 2013.

${ }^{3}$ Code of Federal Regulations, 14 CFR, Part 91, Sec. 91.113 (2004).

${ }^{4}$ Santiago, C., and Mueller, E. R., "Pilot Evaluation of a UAS Detect-and-Avoid System's Effectiveness in Remaining Well Clear," 11th USA/Europe Air Traffic Management Research and Development Seminar, Lisbon, Portugal, June 2015.

${ }^{5}$ Shively, Robert J., Kim-Phuong L. Vu, and Timothy J. Buker. "Unmanned Aircraft System Response to Air Traffic Control Clearances Measured Response." Proceedings of the Human Factors and Ergonomics Society Annual Meeting, vol. 57, no. 1, pp. 31-35, 2013.

${ }^{6} \mathrm{Vu}, \mathrm{K}-\mathrm{PL}$, Gregory Morales, Dan Chiappe, Thomas Z. Strybel, Vernol Battiste, Jay Shively, and Timothy J. Buker. "Influence of UAS pilot communication and execution delay on controller's acceptability ratings of UAS-ATC interactions." Digital Avionics Systems Conference (DASC), 2013 IEEE/AIAA 32nd, pp. 6D4-1, 2013.

${ }^{7}$ Rorie, R. Conrad, and Lisa Fern. "UAS Measured Response The Effect of GCS Control Mode Interfaces on Pilot Ability to Comply with ATC Clearances." Proceedings of the Human Factors and Ergonomics Society Annual Meeting, vol. 58, no. 1, pp. 64-68, 2014.

${ }^{8}$ Calhoun, Gloria L., Crystal A. Miller, Thomas C. Hughes, and Mark H. Draper. "UAS Sense and Avoid System Interface Design and Evaluation." In Proceedings of the Human Factors and Ergonomics Society Annual Meeting, vol. 58, no. 1, pp. 2125, 2014.

${ }^{9}$ Fern, L., Susan R. Flaherty, R. Jay Shively, and Terry Turpin. "Airspace deconfliction for UAS operations." Proceedings of the 16th International Symposium on Aviation Psychology, Dayton, OH, May, pp. 3-5, 2011.

${ }^{10}$ Fern, Lisa, and Jay Shively. "Designing airspace displays to support rapid immersion for UAS handoffs." Proceedings of the Human Factors and Ergonomics Society Annual Meeting, vol. 55, no. 1, pp. 81-85, 2011.

${ }^{11}$ Friedman-Berg, Ferne, Jonathan Rein, and Nicole Racine. "Minimum Visual Information Requirements For Detect and Avoid in Unmanned Aircraft Systems." Proceedings of the Human Factors and Ergonomics Society Annual Meeting, vol. 58, no. 1, pp. 54-58, 2014. 
${ }^{12}$ Draper, Mark H., Jessica S. Pack, Sara J. Darrah, Sean N. Moulton, and Gloria L. Calhoun. "Human-Machine Interface Development for Common Airborne Sense and Avoid Program." Proceedings of the Human Factors and Ergonomics Society Annual Meeting, vol. 58, no. 1, pp. 44-48, 2014.

${ }^{13}$ Bell, S., Jill Drury, Steven Estes, and C. Reynolds. "GDTI: A ground station display of traffic information for use in sense and avoid operations." In Digital Avionics Systems Conference (DASC), 2012 IEEE/AIAA 31st, pp. 8A2-1. IEEE, 2012.

${ }^{14}$ Feitshans, Gregory L., Allen J. Rowe, Jason E. Davis, Michael Holland, and Lee Berger. "Vigilant spirit control station (VSCS) - 'The face of COUNTER'." In Proceedings of AIAA Guidance, Navigation and Control Conf. Exhibition, 2008.

${ }^{15}$ Johnson, Walter W., Vernol Battiste, and Sheila Holland Bochow. A cockpit display designed to enable limited flight deck separation responsibility. No. 1999-01-5567, 1999.

${ }^{16}$ JADEM

17 Prevot, Thomas. "Exploring the many perspectives of distributed air traffic management: The Multi Aircraft Control System MACS." In Proceedings of the HCI-Aero, pp. 149-154, 2002.

${ }^{18}$ RTCA. "Minimum operational performance standards (MOPS) for aircraft surveillance applications (ASA) system". RTCA Inc., Washington, DC, 2011.

${ }^{19}$ Hart, Sandra G., and Lowell E. Staveland. "Development of NASA-TLX (Task Load Index): Results of empirical and theoretical research." Advances in psychology 52, 139-183, 1988. 